Kairos. Journal of Philosophy \& Science 22, 2019

Center for the Philosophy of Sciences of Lisbon University

\title{
The Role of Intuition and Formal Thinking in Kant, Riemann, Husserl, Poincare, Weyl, and in Current Mathematics and Physics
}

"Mathematics is a very human exploration of the patterns of the world, one which thrives on play and surprise and beauty".

(Hermann Weyl, 1949)

"Many people have an impression that mathematics is an austere and formal subject concerned with complicated and ultimately confusing rules for the manipulation of numbers, symbols and equations, rather like the preparation of a complicated income tax return. Good mathematics is quite opposite to this. Mathematics is an art of human understanding... [...] A given mathematical concept might be primarily a symbolic equation, a picture, a rhythmic pattern, a short movie - or best of all, an integrated combination of several different representations".

(William Thurston, 2009)

Luciano Boi

Ecole des Hautes Etudes en Sciences

Sociales, Centre de Mathématiques

Iboi@ehess.fr

DOI 10.2478/kjps-2019-0007

Abstract. According to Kant, the axioms of intuition, i.e. space and time, must provide an organization of the sensory experience. However, this first orderliness of empirical sensations seems to depend on a kind of faculty pertaining to subjectivity, rather than to the encounter of these same intuitions with the real properties of phenomena. Starting from an analysis of some very significant developments in mathematical and theoretical physics in the last decades, in which intuition played an important role, we argue that nevertheless intuition comes into play in a fundamentally different way to that which Kant had foreseen: in the form of a formal or "categorical" yet not sensible intuition. We show further that the statement that our space is mathematically three-dimensional and locally Euclidean by no means follows from a supposed a priori nature of the sensible or subjective space as Kant

O Open Access. (C) 2019 Luciano Boi, published by Sciendo. (cc)BY-NC-ND This work is licensed under the Creative Commons Attribution-NonCommercial-NoDerivatives 4.0 License. 
claimed. In fact, the three-dimensional space can bear many different geometrical and topological structures, as particularly the mathematical results of Milnor, Smale, Thurston and Donaldson demonstrated. On the other hand, it has been stressed that even the phenomenological or perceptual space, and especially the visual system, carries a very rich geometrical organization whose structure is essentially non-Euclidean. Finally, we argue that in order to grasp the meaning of abstract geometric objects, as $n$-dimensional spaces, connections on a manifold, fiber spaces, module spaces, knotted spaces and so forth, where sensible intuition is essentially lacking and where therefore another type of mathematical idealization intervenes, we need to develop a new form of intuition.

Keywords. Intuition, imagination, formalism, axiomatization, space, knot, dimension, non-Euclidean geometries, Kant, Riemann, Husserl, Poincaré, Weyl.

\section{Comments on the Role of Intuition according to Kant}

Kantian philosophy is generally associated with his theory that intuition is, although not exclusively, at the foundation of knowledge. Now, it is true that philosophical thought reached a turning point with Kant in the sense that he showed that intuition could not be developed from one source alone: as was the case with Descartes, who opposed reason and sensibility, or as has been the case with Hume and the English empiricists who limited themselves to the experiences of the senses.

Kant rejected this alternative as irrelevant and in so doing, opened up a new horizon for philosophical research. He helped to show the complex and "dynamical" character of knowledge, as well as the fact that intuition plays an essential role in settling knowledge. The reasons for this are two-fold. Firstly, there are the pure forms of sensibility (or intuition) of space and time, whose function is to order and to yield meaningful the empirical phenomena (the flow of sensations) from which a preliminary legalization may be established, in accordance with what Kant called the axioms of intuition.

One such axiom which Kant presented as being a self-evident (and this, as we will later see, is where the problem lies), asserts that our sensible (or perceptual) space - or in other words, our Euclidean surrounding space and not the abstract mathematical object itself $R 3$ - must have three dimensions. The fact that we recognize that our space has three dimensions and not more (recall that according to general relativity space-time has four dimensions, and that according to recent physical theories such as supergravity and string theory space-time might have 
ten dimensions) means, in Kant's mind, that we must adopt such a postulate in order to begin to make sense of the multitude of incoherent, empirical perceptions and external phenomena. This is the first function which Kant attributes to the intuitions of space (for the external sensibility) and time (for inner sensibility): provide a rational and structural coherence to the multitude of empirical phenomena, a coherence which would, nevertheless, at this point, seem to be less dependent on the internal structure of the real phenomena than on a kind of faculty inherent to subjectivity. In other words, intuition, in this sense, acts as a system of reference, or as a sort of ordering criterion.

The mathematician Bernhard Riemann expressed this Kantian idea in somewhat different, yet more precise terms:

"The hypothesis that space is an infinite and three-dimensional manifold is a hypothesis which applies to our whole perception of the external world, and which allows us, at every instant, to complete the realm of our actual perceptions and construct the possible places of objects; in fact, this hypothesis is constantly confirmed in all of these applications. (...) But the infinitude of space is by no means a necessary consequence of what precedes (Riemann 1990)".

This passage from Riemann demands further comments, since here a new element emerges which is very important when considered in relation to the Kantian position, and which belongs to a question which is still debated today: this concerns the status of intuition in mathematics and physics, and first of all the intuition of space. The very development of these two branches of science, as well as a number of important results obtained throughout the 19th and 20th centuries, have showed that intuition plays a fundamental role in the elaboration of theoretical concepts and in the search for the interaction of the latter with a possible objective knowledge of phenomena. However, this has required a substantial rethinking of our concept of intuition and the role that it can play in our theoretical and real knowledge of the world. 
With reference to this, we can characterize a certain number of theories of knowledge in relation to the status that they each attribute to intuition. Here, I shall restrict myself to consider especially the Kantian conception.

(i) Kant and Kantianism have ascribed to intuition a central role in the entire system of knowledge. In fact, according to Kant, true knowledge cannot exist without intuition, because we can only make sense of external phenomena if they are related to a pure form of intuition which is established a priori - namely the intuition of space and time. More specifically, Kant characterizes the mathematical and theoretic-physical knowledge by the two following properties (or axioms): (1) Mathematical knowledge is founded on intuition and not on concepts, (2) Mathematical knowledge is synthetic and a priori as opposed to analytical in nature.

We would try now to analyze the meaning of these two Kantian axioms, in order to emphasize the points of interest as well as their limits. Firstly we notice that Kant includes geometry as well as arithmetic in the realm of mathematics, giving them essentially the same status, a position which poses some serious problems. Let's consider just one example. We know that the Riemann's zeta function' can be written as a purely formal (arithmetical) expression. In this sense, its definition does not require intuitive reasoning whatever, but a purely mathematical reasoning involving the introduction of a certain number of formal properties which must, first of all, be exactly defined and then rigorously

1 The Riemann hypothesis is a famous deep mathematical conjecture first stated by Riemann first in the groundbreaking 1859 paper "Über die Anzhal der Primzhalen unter eine gegebenen Grösse", where the great mathematician obtained an analytical formula for the number of primes up to a preassigned limit. The formula is expressed in terms of the zeros of the zeta function, namely the solutions $p \in \mathbf{C}$ of the equation $\mathrm{V}(p)=0$. In its very clear account, Bombieri wrote [2000, p. 1], «The Riemann zeta function is the function of the complex variable $s$, defined in the half-plane $R(s)>1$ by absolutely convergent series

$\varsigma(s):=\sum_{n=1}^{\infty} 1 / n^{s}$,

and in the whole complex plane $\mathbf{C}$ by analytic continuation. As shown by Riemann, $\mathrm{V}(s)$ extends to $\mathrm{C}$ as a meromorphic function with only a simple pole at $s=1$, with residue 1 , and satisfies the functional equation

$\pi^{-s / 2} \Gamma(s / 2) \varsigma(s)=\pi^{-(1-s) / 2} \Gamma(1-s / 2) \varsigma(1-s)$.

The statement that all zeros of the function $\mathrm{V}(s)$ are real is the Riemann hypothesis". 
proved. From that point of view, it would be worthless try to assign any kind of intuitive content to these formal properties. It is much more in the abstract ideas, the formal generative techniques and the reasoning based on the symbolic mathematical structures, which we need to look for the source of arithmetic thought.

This does not, however, mean that intuition plays no role at all; for intuition comes into play in a fundamentally different way to that which Kant had foreseen: more precisely, in the form of an intellectual (or "categorial" in Husserl's terms) and not sensual intuition, this being an active and creative intuition, as opposed to a passive and receptive intuition. In mathematics and theoretical physics, the intuition is often related with the introduction of a profound idea which reveal a new link among different fields of mathematics or of a deep conjecture, which open a new landscape in a given field of research.

\section{Some Remarks About the Role of Intuition and Conceptualization in Modern Mathematics and Physics}

The previous remarks should help to understand better why certain arithmetical invariants recently discovered in the framework of research, in algebraic geometry, on the four-dimensional and compact manifolds or in knot theory, are directly linked notably with the geometric and topological structure of these same mathematical objects; or in other words, how algebraic and topological invariants such as the Alexander or Donaldson invariants, hold an enormous amount of information on both the local (differential) and global (topological) behavior of a large class of mathematical objects which have, until now, been considered separately. Let us now consider in more detail these two examples, for which it is very important to underline the following facts.

\subsection{Some Mathematical and Physical Developments}

(i) The Alexander polynomial of a knot or a link, which has become one of the cornerstones of knot theory, is very closely connected with the topological properties of the knot. Also of great significance is that there are various methods by which we may calculate the Alexander polynomial; one of these methods is called the Seifert matrix. Concisely, 
"the Seifert matrix of a knot is a very combinatorial topological and very nice tool allowing to show how various surfaces can be constructed from the same knot diagram: it suffice that the linking numbers for all these surfaces be equal or rearranged in such a way that they turn out to be equivalent. He described a method for associating with each knot a polynomial such that if one form of a knot can be topologically transformed into another form, both will have the same associated polynomial. Now, suppose $M$ is the Seifert matrix of a knot (or link) $K$, then

$\operatorname{det}(M+M T)$

is an invariant of the knot $K$. Furthermore, the determinant of a knot (or link) $K$ is completely independent of the orientation assigned to $K^{\prime \prime}$ (Murasugi 1996, 76).

It is here important to underline that the Alexander polynomial arises from the homology of the infinite cyclic cover of the complement of a knot. Equivalently it can be derived from considering cohomology of the knot complement with coefficients in a flat line-bundle. Following Kauffman, we can now define mathematically, in a summarized way, the Alexander polynomial.

"Let $K \subset S^{3}$ be an oriented knot or link, and $F \subset S^{3}$ a connected oriented spanning surface for $K$. Let $\theta: H_{1}(F) \times H_{1}(F) \rightarrow Z$ be the Seifert pairing.

Definition 1. Two polynomial $f(t), g(t) \in Z(t)$ are said balanced (written $f=g$ ) if there is a non-negative integer $n$ such that $\pm t^{n} f(t)=g(t)$ or $\pm t^{n} g(t)=f(t)$.

Definition 2. Let $K, F, \theta$ be as above. The Alexander Polynomial, $\Delta_{K}(t)$, is the balance class of the polynomial $\Delta_{K}(t)=$ $D\left(\theta-t \theta^{\prime}\right)$. It follows from $S$-equivalence (due to Seifert) that this determinant is well defined on isotopy classes of knots and links up to multiplication by factors of the form $\pm t^{n \text { " }}$ (Kauffman 1987, 229). 
Finally, we recall that the topologist Herbert Seifert established an important result in 1927 according to which there can be different surfaces for isotopic knots. Stated differently, a given knot or link can have many various spanning surfaces. For example, two isotopic diagrams will have rather different Seifert surfaces.

(ii) In short, "Donaldson's result implies that there exist "exotic" 4-dimensional spaces which are topologically but not differentially equivalent to the standard Euclidean 4-space $R^{4}$. This is a purely mathematical fact. But there is another fact no less important and intimately related to the first one, namely the Donaldson's results are derived from the YangMills equations of theoretical physics, which are non-linear generalizations of Maxwell's equations" (Atiyah 1986, 3-4; 2011, 1986).

This example proves that mathematical concepts can be rooted, in some way, in physical theories, or, to put it in other words, that the physical insights concerning the most striking properties of some kind of phenomena may suggest very important progress in mathematics, and especially lead to discover some mathematical structures that are supposed to be closely related to the physical phenomena, which one is struggling to describe and to explain. In fact, there are interesting situations which shows that the meant similarity between ideas about physical phenomena and purely mathematical structures has led to a further deepening and in some cases to an axiomatization of entire fundamental field of mathematics.

For instance, the recent Topological quantum field theory ${ }^{2}$ is a very good example that shows how one can "translate" a number of physical insights into precise and significant mathematical statements. Clearly a great deal of "philosophical" imagination and "mathematical" intuition are necessary in order to carry out such a translation. The starting point was the recognition of a link between quantum physics and topology and therefore of the fact that global aspects are much more important than the purely local aspects. Thus,

"quantum field theory is viewed as the differential geometry of certain infinite-dimensional manifolds (spaces

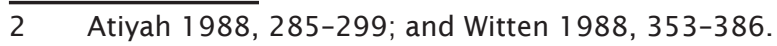


of connections), including the associated analysis (e.g. Hodge theory) and topology (e.g. Betti numbers). One must add that the quantum field theory is inherently non-linear, and this non-linearity comes from non-Abelian Lie groups. Moreover the fundamental topological aspects of such a quantum field theory should be independent of the scaling or coupling parameters. This means that such topological information is essential independently of the analytical details of the quantum theory. That is why such topological understanding is a necessary pre-requisite to building the analytical apparatus of the quantum theory. The next step is to construct axiomatically this theory in order to formulate its essential structural features. Obviously such a construction is of interest independently of any physical interpretation" (Atiyah 1988, 175-6).

Atiyah proposed an axiomatic construction of topological quantum field theory (Atiyah 1988, 178).

"A topological quantum field theory in dimension $d$ defined over a ground ring $\Lambda$, consists of the following data: (i) A finitely generated $\Lambda$-module $Z(\Sigma)$ associated to each oriented closed smooth $d$-dimensional manifold $\Sigma$,

(ii) An element $Z(M) \in Z(\partial M)$ associated to each oriented smooth $(d+1)$-dimensional manifold (with boundary) $M$.

These data are subject to the following axioms:

(a) $Z$ is functorial with respect to orientation preserving diffeomorphism of $\Sigma$ and $M$,

(b) $Z$ is involutory, i.e. $Z\left(\Sigma^{*}\right)=Z(\Sigma)^{*}$ where $\Sigma^{*}$ is $\Sigma$ with opposite orientation and $Z(\Sigma) *$ denotes the dual module, (c) $Z$ is multiplicative.

The first axiom means that an orientation preserving diffeomorphism $f: \Sigma \rightarrow \Sigma^{\prime}$ induces an isomorphism $Z(f)$ : $Z(\Sigma) \rightarrow Z\left(\Sigma^{\prime}\right)$ and that $Z(g f)=Z(g) Z(f)$ for $g: \Sigma^{\prime} \rightarrow \Sigma^{\prime \prime}$. Also if $f$ extends to an orientation preserving diffeomorphism $M \rightarrow M^{\prime}$, with $\partial M=\Sigma, \partial M^{\prime}=\Sigma^{\prime}$, then $Z(f)$ takes $Z(M)$ to $Z\left(M^{\prime}\right)$. 
The meaning of the second axiom is clear when $L$ is a field in which case $Z(\Sigma)$ and $Z(\Sigma)^{*}$ are dual vector spaces. This is the most important case and, for physical examples $\Lambda=\mathbf{C}$ (or perhaps $\mathbf{R}$ ). However, there are interesting examples with $\Lambda=Z$. In this case the relation between $Z(\Sigma)$ and $Z\left(\Sigma^{*}\right)$ is like that between integer homology and cohomology. The duality can be formalized by considering free chain complexes. But instead we may take $L$ to be a field and the case $\Lambda=\mathbf{Z}$ can essentially be replaced by the fields $\mathbf{Q}, \mathbf{Z} p$.

The multiplicative axiom asserts first that, for disjoint unions, we have $Z\left(\Sigma_{1} \cup \Sigma_{2}\right)=Z\left(\Sigma_{1}\right) \otimes Z\left(\Sigma_{2}\right)$.

Moreover if $\partial M_{1}=\Sigma_{1} \cup \Sigma_{3}, \partial M_{2}=\Sigma_{2} \approx \Sigma^{*}{ }_{3}$ and $M=M_{1} \cup \Sigma_{3} M_{2}$ is the manifold obtained by gluing together the common $\Sigma_{3}$-component, then we require:

$$
Z(M)=\left\langle Z\left(M_{1}\right), Z\left(M_{2}\right)\right\rangle \text {. }
$$

Where $\langle$, $\rangle$ denotes the natural paring

$$
Z\left(\Sigma_{1}\right) \otimes Z\left(\Sigma_{3}\right) \otimes Z\left(\Sigma_{3}\right) * Z\left(\Sigma_{2}\right) \rightarrow Z\left(\Sigma_{1}\right) \otimes Z\left(\Sigma_{2}\right) \text {. }
$$

It is here worth mentioning the intimate interaction highlighted in the last three decades, which is of fundamental importance both for mathematics and theoretical physics, between non-Abelian gauge theory and the algebraic and topological theory of four-dimensional compact manifolds. Actually, in this example there is more than an analogy between physical properties of phenomena (in most the cases, theoretically stated but not necessarily empirically observed) and mathematical structures and invariants.

Still a few words on this subject are in order. The starting point for Donaldson's work is Yang-Mills theory, an example of non-Abelian gauge theories (see Donaldson 1983, and Witten 1988). Yang-Mills is defined on a four-dimensional manifold $M$, and the action is given by $A \rightarrow \int_{\mathrm{M}}\left\|F_{A}\right\|^{2}$

where $A$ is a connection with curvature $F_{A^{*}}$. The action is minimized by instantons, which are solutions to the self-dual Yang-Mills equations. 
One can associate to an instanton an integer $k$ known as the charge, and study the moduli space of instantons of charge $k$. When $M$ is a complex algebraic surface this can be described using algebraic geometry. Using moduli spaces, Donaldson defined invariants of the manifold $M$. One crucial point is to show that they are invariants of the smooth structure and do not depend on the choice of Riemannian metric on $M$.

We may now compare Yang-Mills theory to electromagnetism. Both are gauge theories, but in Yang-Mills the non-Abelian nature of the group (the non-commutative compact Lie group SU(2)) leads to non-linear equations. The most remarkable fact is that in this theory a metric is used in the definition of the fundamental equations, but the invariants produced are metric-independent. It is now natural to ask if there is a physical interpretation of the Donaldson invariants. Edward Witten showed in the late 1980's that Donaldson theory might be regarded as an $N=2$ twisted supersymmetric Yang-Mills quantum field theory (see Witten, 1988). Here the "twisting" involves changing the spin of certain particles, and make the theory into a metric-independent topological field theory. We saw thus that these invariants reveal themselves to be very important in order to elucidate certain aspects of gauge theories.

On the other hand, fundamental results obtained by P. Kronheimer and T. Mrowka (1994) showed that,

"starting with a gauge-theoretic treatment of surfaces in four-manifolds, one is able to found recurrence relations for the Donaldson invariants, and to show that, for a large class of four-manifolds $M$, the generating function for these invariants could be expressed in terms of the intersection form of $M$, together with a finite number of basic classes in $H^{2}(M)$. Recent progress in this field has involved the notion of duality. One early example is the duality between electricity and magnetism in Maxwell's theory. A more sophisticated kind of duality was conjectured by $\mathrm{C}$. Montonen and D. Olive in 1977 to hold for non-Abelian gauge theories. They suggested that, given a Yang-Mills-Higgs gauge theory with group $G$, one could form a dual theory with a "dual" group $G^{*}$, in such a way that gauge particles mediat- 
ing physical forces in one theory correspond to monopoles in the dual theory, and vice versa. This duality interchanges electric charges (related via Noether's theorem to the existence of symmetries), and magnetic charges, which are topological in nature (related to the degree of the Higgs field), that is the maximal tori of $G$ and its dual group in the usual sense of tori" (Atiyah 1966, 5-6).

Let us briefly consider another important example, which is closely related to the above. "One of the great mathematical advances in this century was the introduction of characteristic classes by Whitney and Stiefel in 1935, characteristic forms by Pontrjagin (over a real fiber space) and Simons-Chern forms (over a complex space) some years later. Intuitively, these "objects" are geometrical and topological invariants that can be classified in different families even though they are all mutually related. Thus, we can topologically transform a surface (or a manifold) into another if they have the same characteristic invariants (and if the dimension of space is compatible with the type of transformation). These invariants and their corresponding algebraic structures can be technically very complicated. The two invariants mentioned above globally characterize the mathematical objects of fiber spaces and connections, which in turn imply several basic algebraic and geometric notions such as homology (homology group, intrinsic homology, singular homology, algebraic homology, functors, etc.), cohomology classes, homotopy, etc. Two examples of homology and cohomology that are very important in contemporary physics (and especially in topological quantum field theory) are bordism and cobordism as developed by René Thom, John Milnor and others, and ordinary homology $H(X)$ (see Bennequin (2001)). In fact, several notions of classical field theory can be expressed by cohomology. The most recent quantum field theories, re-interpreted in the mathematical framework of gauge theory, show a massive presence of cohomological ideas, seen in some cases as a generalization of characteristic classes such as those of Euler-Poincaré. A very interesting example in our time is that of a non-Abelian cohomology space of Riemannian surfaces or manifolds with boundary" (Boi 2004b, 1788). 
For each topological space $X$, the commutative group $\Omega(X)$ can be defined as follow.

"Continuous maps $f: Y \rightarrow X$ from oriented and compact manifolds with boundary $Y$ in $X$ are called chains. Disjoint union and change of orientation define the sum and difference of chains respectively. The boundary of $X$ is its restriction to the boundary of $Y$. It is well-known (by a fundamental theorem of algebraic geometry) that the boundary of a boundary is empty: $\partial \cdot \partial=0$. A cycle is a chain whose source has non-boundary. The equivalence classes of cycles form the group $\Omega(X)$. The Thom ring is the bordism of a point. With the product $Y \times X$ one can see that $\Omega(X)$ is a module over $\Omega$, so that $\Omega$ acts on $\Omega(X)$. To every continuous map $X_{1} \rightarrow X_{2}$ is associated a linear transformation of $\Omega\left(X_{1}\right)$ into $\Omega\left(X_{2}\right)$; we have then a functor. The cobordism cohomology $\Omega^{*}(X)$ is obtained by taking the homotopy classes of continuous maps of $X$ into a given space, in fact a nested sequence of topological spaces, the Thom spectrum. The cohomology (the theory $\Omega^{*}$ ) is richer than the homology $(\Omega)$, dealing with rings having all sorts of operations. Cobordism is an equivalence relation on the set on submanifolds, say $N$ and $N$ ' of $M$; which means that the cobordism $Z$ transform $N$ into $N^{\prime}$. M designate a compact oriented manifold with $H_{1}(M)=0$. We say then that two submanifolds $N$, $N^{\prime}$ are cobordantes in $M$ if there is a compact $Z=M \times[0,1]$ so that $\left.\partial Z=N \times\{0\} \subseteq N^{\prime} \times\{1\}\right\}^{\prime \prime}$ (Boi 2004, 1792-93).

These brief considerations serve to highlight some basic characteristics of cohomology that make it a good basis for building a richer and more subtle theory of the spatial continuum and of space-time, with significant theoretical and philosophical consequences for physics. Some of these characteristics are: (i) Homology is constructed by quotienting a part of the data (cut and gluing). It stabilizes forms. (ii) It shows the close relationship that can exist between figures and numbers, especially coefficients. We can reconstruct a new ring $\Omega$ from combinations of chains with rational $(\mathbf{Q})$ or complex coefficients $(\mathbf{C})$. This allows us to "localize" 
and to "complete" respectively. (iii) Its most remarkable property is universality. There are many cohomologies that give the same results. More precisely: different definitions lead to isomorphic (or related, at the very least) theories. This means that axiomatic constructions are conceivable. (iv) Cohomology engenders forms; in some sense, it defines forms. In any case it ensures a certain stability and genericity. Several notions from classical field theory can be expressed cohomologically. Furthermore, the most recent quantum field theories, re-interpreted in the mathematical framework of gauge theory, highlight the basic role played by cohomology and characteristic classes. These concepts are also utilized in the attempts to give a consistent mathematical formulation and an intelligible physical interpretation of other gauge quantum theories such as the quantum electrodynamics of Dirac, Feynman and Schwinger.

\section{The Kant's Conception of Space, and Some Mathematical Results}

The last remarks are of interest to our discussion of the Kantian conception of mathematics as well as of intuition. Kant has argued: (1) First (in the Transcendental Aesthetic), that the mathematical knowledge is based upon intuition rather than upon concepts, and that space and time are nothing else but pure forms of our sensibility which are necessary in order to organize the variety of very different unrelated empirical sensations, yet still unable to yield knowledge of phenomena; and (2) Second (in the Transcendental Analytic), that the formal determinations (or pure intuitions) of space and time enable the anticipation of phenomena, because they provide an a priori representation of these same phenomena, which can be found a posteriori to exist empirically.

Of course, here we are concerned with the distinction made by Kant between the metaphysical nature of space and time and their transcendental character. From the metaphysical point of view, space and time are schemes and functions of our sensibility or subjectivity at once. Furthermore, Kant states that they are necessary and a priori. Finally, he derives from the previous two features the existence of certain axioms of geometry as well as their apodictic validity.

Recall two of these axioms. The first states that the straight line - with the properties ascribed to it by Euclid - is the shortest path joining any 
two points in the plane. The second say that our space must necessarily have three dimensions. Now, we already saw talking about Riemann, that the 3-dimensional feature we ascribe to our space is much more a hypothesis allowing for localizing the objects of perception in the surrounding space than a necessary and a priori property inherent to space. Of course, anyone would deny the fact that our space is, mathematically, 3-dimensional and that it is locally Euclidean, but such a statement by no means follows from a supposed a priori nature of the sensory or subjective space, whatever it could be. On the contrary, this statement has the following two meanings:

(i) The postulate asserting that space has three dimensions is mathematical in nature and it is obtained by idealization from the objective properties of things and bodies "living" in the surrounding (Euclidean) space. In other words, it means that we are able to localize and represent these same things and bodies using a three-values system of coordinates or a system of three continuous and linear functions. Moreover, we ascribe to such a space some fundamental properties like homogeneity, isotropy and infinity. Notice however that all these properties are essentially metrical in nature, for actually topologically the things turn to be different. In fact there exist 2- or 3-dimensional spaces that are locally Euclidean, yet globally non-Euclidean. For example, the Clifford surface, whose metrical relations are the same as those that characterize the elliptical (Riemannian) space, fulfills nevertheless the principal axioms of Euclidean geometry, i.e., it can be conformally mapped into small regions of the Euclidean plane. A parallelogram over a Clifford surface enjoys the following properties: (a) The straight lines of the Euclidean plane are represented by geodesics, (b) The sum of angles of a Euclidean triangle is equal to $2 \pi$, (c) The curvature is everywhere constant and equal to 0 .

The Clifford parallelogram is formed as follows.

"Given any two intersecting lines, the left parallel to one through any point of the other meets the right parallel to the latter through any point of the former. The quadric generated by the above systems of lines is called a Clifford surface. By a famous theorem of Gauss, the "total curvature" of a geodesic polygon on any surface is measured by its angular 
excess (as compared with a Euclidean polygon of the same number of sides). Since a Clifford surface is covered with a network of Clifford parallelograms, as small as we please, it follows that this surface has zero curvature everywhere, which means that it can be mapped without distortion on a suitable region of the Euclidean plane. To use a nice picture, a small creature whose world was a Clifford surface would find his practical geometry to be Euclidean, until he explored so far as to discover that this "flat earth" was finite (though unbounded). In other words, after cutting the Clifford surface along any two intersecting generators, we can "develop" it on the Euclidean plane, just as we can unroll a cylinder. The Clifford parallelogram, and the whole surface will appear as the interior of a rhombus of side $\pi$ and angle $y$. Thus its total area is $\pi^{2} \sin \psi$. A Clifford surface is a surface of revolution in two distinct ways. It follows that the geodesics $x-y=c$ are circles (of the same radius for all values of $c$ ), and likewise the geodesic $x+y=c$. When the surface is developed on the Euclidean plane, two such circles, one of each kind, appear as diagonals of the rhombus. Hence, by Euclidean trigonometry, their circumferences are $2 \pi \sin 1 / 2 \psi$. In the special case of a rectangular Clifford surface, the rhombus is a square, and the circles are equal. A Clifford surface, being closed and uniform like a sphere, developable like a cylinder, and ring-shaped like a torus, is no easy to visualize. Unlike a sphere, it has zero curvature; moreover, in the rectangular case, its "inside" and "outside" are congruent. Unlike a cylinder, it is finite, and has two systems of generating lines" (Coxeter 1988, 143-45).

(ii) The second meaning above has manly a physical and physiological content. It entails the fact that the possibilities of movement in our 3-dimensional space are fairly limited. In fact, we can essentially move only in three directions; however, since we need much more free mobility for our displacements in space, we are forced to introduce some complicated geometrical objects and operations. That is one of the reasons 
because the three- and four-dimensional manifolds have a very complex geometrical and topological structure which still resists to a fully understanding, in spite of the outstanding results obtained by Steven Smale in 1958.

Roughly, Smale discovered that the manifolds of greater dimension than $4(\geq 4)$ are relatively easily to understand; he proved the Poincaré's conjecture for $n=5$. The Poincaré's conjecture states that the only 3-dimensional closed and simply connected manifold is the topological sphere S3; some time later, these conjecture was generalized to manifolds of any dimension. Another outstanding result was obtained by Michael Freedman, who gave the complete classification of 4-dimensional simply connected topological manifolds; this hold as proof of the generalized Poincaré's conjecture in 4-dimensions. More precisely, Freedman has proved that any quadratic form $Q$ is the intersection form of a certain topological closed and simply connected manifold $V^{4}$, which show that it may exist a very intimate relationship between algebra and topology. There has been finally the very fundamental result by Simon Donaldson in 1983, which showed, on the one hand, that relatively few 4-dimensional topological manifolds discovered by Freedman possesses a differentiable structure, on the other hand, that the world of differentiable 4-dimensional manifolds is much more richer and deeper than that which characterize the other dimensions. For example, $R^{4}$ possesses infinity of differentiable structure called "exotic". In more technical words, Donaldson has first showed that, in the differentiable framework, there are strong constraints so that an integer unimodular quadratic form can be an intersection form. This completely contrasts with the topological situation where there is none of such restrictions. The result of Donaldson shows after all that the distinction topological/differentiable is very hard to understand in 4-dimensions and maybe still more complicated in 3-dimensions.

These results are very important for a deeper philosophical reflection about the nature of space and the kind of structures that it can support. In fact, they push far beyond the possibilities of a new inquiry into the mathematical as well as ontological meaning of space. In particular, they shows that one can conceive and prove the mathematical existence of many kinds of spaces whose abstract properties does not share nothing 
with those properties we generally ascribe to our sensory space. Recall some basic facts we had learned in the last half-century from topology and differential topology.

(i) The same space (or manifold) can bear different geometrical structures; this is especially true for $R^{3}$ and $R^{4}$. Now it is clear that this fact contradict one of the most important assumptions of the Kant's theory of space and geometry (as formulated in the Critique of Pure Reason, "Transcendental Æsthetic"), namely the uniqueness of the geometrical structure of our usual Euclidean space, whose most essential properties can be stated by a set of very simple axioms which pertain to intuition but not to concepts. (ii) Two spaces (or manifolds) can be homeomorphic (topologically equivalent) although not diffeomorphic (differentially equivalent). An important theorem states that there exists an exotic $R^{4}$ which is homeomorphic to, but not diffeomorphic to $R^{4}$. (iii) Two or more inequivalent manifolds can carry the same intersection form.

"In order to explain the important notion of intersection form, consider one of the classical results of topology, that is the classification of compact connected surfaces (without boundary) up to diffeomorphism. Let $\Sigma$ be such a surface and consider closed curves $\gamma_{1}$ and $\gamma_{2}$ on $\Sigma$. By a small deformation we can make $\gamma_{1}$ transversal to $\gamma_{2}$. The curves then intersect in a finite number of points. This number, modulo 2 , turns out to depend only on the homology class of $\mathrm{g}_{1}$ and $\gamma_{2}$ in $H_{1}\left(\Sigma ; \mathbf{Z}_{2}\right)$. We thereby get a symmetric bilinear form $\mu$ on $H_{1}\left(\Sigma ; \mathbf{Z}_{2}\right)$ called the intersection form of $\Sigma$. Poincaré duality says that this form is nondegenerate. One define the form to be of type II if $\mu(x, x)=0$; otherwise, we say it is of type I" (Lawson 1985, 1-2).

As Lawson further showed, from that we get two important theorems which emphasize the point (iii).

Theorem 1. Two compact connected surfaces are diffeomorphic if and only if their intersection forms are abstractly equivalent. Surfaces can be separated into two classes: type I and type II: those of type I are nonorientable and can be decomposed into a connected sum of real projec- 
tive planes. Those of type II are orientable and can be decomposed into a connected sum of tori. To put it into a formulae, we have for the type I - nonorientable surfaces (manifolds) with $\left(w_{1} \neq 0\right)$ and

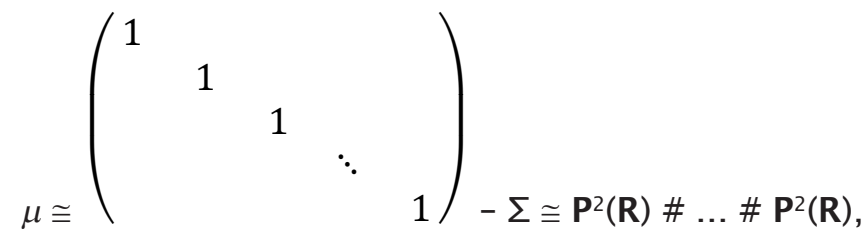

and for the type II - orientable surfaces (manifolds) with $w 1=$ and $\mu \cong\left(\begin{array}{ccc}1 & & \\ & \ddots & \\ & & 1\end{array}\right)-\Sigma \cong\left(S^{\prime} \times S^{\prime}\right) \# \ldots \#\left(S^{\prime} \times S^{\prime}\right)$.

Theorem 2. (J.H.C. Whitehead). Two compact simply-connected 4-manifolds are homotopy equivalent if and only if their intersection forms are equivalent.

(iv) It is clear that (a) there are several kinds of $n$-dimensional spaces or manifolds whose structures my be equivalent, partially equivalent (for example metrically though not topologically), or not at all equivalent; (b) the same space or manifold can carry many different structures, either differentiable or topological; (c) each of these spaces can be characterized by a set of objective mathematical properties, in the sense that they would not depend on our perception or the organization of our inner sensory systems, i.e., on the "subjectivity" as Kant believed. In fact, these objective properties enable us to distinguish between different family of geometrical structures and to say whether two spaces are equivalent or not, and also to recognize the type of equivalence we are dealing with. For example, the connectedness, the orientability or nonorientability, the curvature and so on, are objective properties of space strictly speaking.

Finally, we would like here to mention that the French mathematician and philosopher Henri Poincaré has expressed a different point of view from that stated by Kant. The relevant points of the Poincaré's reasoning can be summed up as follows.

1) First, the sensory space has little in common with geometrical space. This because the space constructed starting from a classification of our sensations according to their physiological sameness is still not a geo- 
metrical space, because it would be neither infinite, nor homogeneous, nor isotropic. As Poincaré stressed,

"Seeing that our representations are simply the reproductions of our sensations, therefore we cannot image geometrical space. We cannot represent to ourselves objects in geometrical space, but can merely reason upon them as if they existed in that space. (...) Geometrical space, therefore, cannot serve as a category for our representations. It is not a form of our sensibility. It can serve us only in our reasoning. It is a form of our understanding" (Poincaré 1898, 21-22).

2) Second, a geometrical space is given once we have properly introduced and defined the concept of group of transformations and its formal properties. In other words, it is the group (and the geometry we associate to it) that yields a certain type of space; moreover the way we make act the group upon a space (or a manifold) generates the characteristic properties of this space. As a modern mathematical translation of this fact, mention for example that the classification of compact simply-connected topological 4-manifolds can be stated in terms of an intersection form on the middle-dimensional homology group. ${ }^{3}$

Now return to the concept of group. First of all, it is important to understand that its formal properties are independent of the qualitative character of the phenomena like different kind of changes affecting their state or their direction. Mathematically, a group is the set of a certain number of operations (including addition, multiplication, associativity, continuity, inverse map, closure, compactness and so forth) and of all combinations which can be made of them. If these operations combine according to the same laws, we then say that the two groups are isomorphic. For example, the different permutations of a certain number of objects, no matter what kind of objects they are, form a group. The formal properties are precisely those which are common to all isomorphic groups.

3 See on this subject S. Eilenberg 1950. 
From what precede, Poincaré was lead to make the distinction between the degree and the order of a group: the first refers to the number of the objects and the second to the number of the permutations. In fact, two groups may be isomorphic and their permutations may combine according to the same laws without their degree being the same. Let us consider the different ways in which a cube can be superposed upon itself. The vertices may be interchanged one with another, as may also be the faces and the edges; whence result three groups of permutations (or of infinitesimal operations) which are evidently isomorphic among themselves; but their degree may be either eight, six, or twelve, since there are eight vertices, six faces, and twelve edges. According to Poincaré, the degree is, so to speak, a material element, and the order a formal element, the importance of which is far greater. We see thus that geometry is not an experimental science; experience forms merely the occasion for our reflecting upon the geometrical ideas which pre-exist in us. Space is but a form of our sensibility, since we cannot represent things to ourselves as they actually are, especially when the dimension of space is greater than three, but we think upon things as if they were embedded or immerged in space. According to Poincaré,

"What we call geometry is nothing but the study of formal properties of a certain continuous group; so that we may say, space is a group. The notion of this continuous group exists in our mind prior to all experience" (Poincaré $1898,41)$.

To better understand what is meant by "formal properties" of a group, we can think of the simplest case of a group, namely the group of Euclidean displacements or the group of all rotations in three-dimensional Euclidean space $S O(3) .{ }^{4}$ Another fundamental example is that of a topological group (see Pontryagin, 1946). In mathematical parlance, it is a

$4 \quad$ Recall that «the group $S O(3,1)$ does for Minkowski space-time or less what $S O(3)$ does for Euclidean space. It is called the Lorentz group. One might think of it as the group of $4 \times 4$ matrices preserving the standard Minkowski metric

$\square(\square, \square)=-\square^{0} \square^{0}+\square^{1} \square^{1}+\square^{2} \square^{2}+\square^{3} \square^{3}$.

It contains the spatial rotations in an obvious way, but also contains the Lorentz transformations that mix up space and time coordinates» (Baez \& Muniain 1994, 163). 
group $G$ endowed with a Hausdorff topology such that the map $G \times G \rightarrow G$ that sends $(x, y)$ onto $x y^{-1}$ is continuous. It follows at once from this definition that the inversion map on $G$ is continuous, because this map can be factored according to the scheme $x \rightarrow(1, x) \rightarrow 1 x^{-1}=x^{-1}$, where 1 stands for the neutral element of $G$. Hence the multiplication is also a continuous map $G \times G \rightarrow G$, because it can be factored according to the scheme $(x, y) \rightarrow\left(x, y^{1}\right) \rightarrow x\left(y^{-1}\right)^{-1}=x y$. In particular, this implies that if $x$ is any element of $G$, then the left and right translations effected by $x$ on $G$, sending an element $y$ of $G$ onto $x y$ or $y x$, respectively, are homeomorphisms of $G$ onto $G$.

The topology of a topological group $G$ is determined by any fundamental system of neighborhoods of 1, i.e., a family of neighborhoods of 1 such that every neighborhood of 1 contains a member of the family. In fact, if $S$ is a fundamental system of neighborhoods of 1 , then a subset $A$ of $G$ is open if and only if, for every point $x$ of $A$, there is a member $V$ of $S$ such that $V_{x} \subset A$. It is convenient to define a topology on a group $G$ by giving a fundamental system of neighborhoods of 1 . A family $S$ of subsets of a group $G$ is a fundamental system of neighborhoods of 1 for a topology with which $G$ is a topological group if and only if the following conditions are satisfied: (i) The intersection of $S$ is the set consisting of 1 alone. (ii) The intersection of every pair of members of $S$ contains a member of $S$. (iii) For every member $V$ of $S$, there is a member $W$ of $S$ such that $W W^{-1} \subset V$. (iv) If $x$ is a point of $G$ and $V$ is a member of $S$ then $x V x^{-1}$ contains a member of $S$.

In light of the previous discussion, the main question which we must now ask is the following:

"why does the number of spatial dimensions play such a important role and why, in particular, is the structure of our space $R^{3}$ and of space-time $R^{4}$ of relativistic physics so difficult to comprehend? The first conceivable answer to this question is that in larger dimensions there is more free space available to us to perform the necessary geometrical constructions, whereas in three dimensions our movements are fairly limited. These constructions do not use complicated objects, just simple discs which are em- 
bedded in the manifold being studied and which do not cut across each other. The existence of such discs is necessary, from a technical point of view, in order to move certain more complicated objects, thus forcing them to be disjoint each from one another: but just as two lines can be separated from each other in the third dimension, two lines (or two surfaces) can always be separated in the fifth (or larger) dimension. On the other hand, in three dimensions, two discs may cut across each other along a line or, in the fourth dimension, at two isolated points" (Baez \& Muniain 1994, 163).

\section{The Role of Intuition in Mathematics and Physics According to Kant and Husserl}

It is not always easy to understand exactly what Kant means when he speaks about intuition. Nevertheless, we can distinguish at least three main aspects of Kant's reasoning.

(1) Firstly, there are sensual or empirical intuitions that are directly associated with our perception of external phenomena, and which affects our sensory system, the latter remaining however unaffected by these external phenomena. Thus, here Kant is implicitly supposing that intuition is nothing more than the sensual content which supplements our different perceptions; however, in such process there is not yet active intervention of the subject on these contents, nor does he construct truly objects of experience starting from these phenomena, which are meant by Kant as given in their immediate appearance and which are hence perceived through our intuition in a passive manner.

(2) Then come the pure intuitions of space and time which are certainly linked to our sensual perceptions, and which organize these perceptions rather than to depend upon them. In other words, these intuitions are not derived from our experience, and there is no direct relationship between the two. On the contrary, what is true for Kant is that our experiences only become objective and meaningful to us when we apply the pure form of intuition, i.e. space and time; outside of these forms of intuition, no experience is possible. 
But what is the precise nature of these intuitions? And how do external phenomena constitute within these intuitions or claim their existence thanks to them? Kant does not seem to put forward any real answer to such questions, which are nevertheless important in order to understand the role of intuition in the knowledge process. If we were to retain one element of Kant's reasoning, we should retain this: intuitions of space and time are qualities which are inherent to, or constitutive of our sensory activity or which are a function of our subjectivity. According to Cassirer, the expression of our subjectivity serves to further underline the active and productive nature of the pure intuitions of space and time and cut the direct ties which link them to the empirical sensations.

Let's stress, at this point, that actually certain spatial dimensions of an object, as well as a good number of their geometric properties, cannot be represented intuitively. Still a direct perception of 3-dimensional objects does, in most situations, present some serious difficulties, for which we need, if we are to overcome them, to use certain geometric notions which supply for our immediate and direct intuition.

It was notably Husserl, in Thing and Space (Lectures of 1907), who showed how the constitution of physical objects, such as spatial objects falling within our field of perception, require the execution of a number of operations and gestures to render the geometric properties that can be virtually transferred to the objects by means of "intuitive filling-in" (anschaulich Erfüllung), according the Husserl's expression. This intuitive completion is, of course, a consequence of the fact that any subject, starting with its body, is able to execute operations which obey certain geometrical movements such as rotations, or different kinds of movement such as ocular, motor or kinesthetic movements, etc. These schema, although being founded on their own laws and abstract geometric properties, and hence a priori in this sense; cannot become truly effective unless they are in concreto involved in the constitution of spatial objects.

When we pass on to a productive thinking of ideal geometric objects as, for example, $n$-dimensional space, connections, fiber spaces, module space, categories, and numerous other algebraic and topological structures which belong specifically to modern mathematics, and where sensual intuition is entirely lacking, another type of mathematical imag- 
ination and visualization is required, and therefore we need to look for a deeper form of intuition.

\section{The Difference Between Sensual Intuition and Mathematical Intuition: From Surfaces to Knot Theory}

Let us make a few reflections about some aspects of this important issue. First, we propose to distinguish between the perception of categories (or schema) of forms and the perception of forms. The objects that fall into the second category can be simply seen (although, of course, the vision process is a complex and multimodal one), whereas the objects which fall into the first category cannot be simply and directly seen, but visualized or imagined. Thus the second kinds of objects cannot be "seen" by our usual visual abilities, for, in fact, they require a sort of schematic and imaginative vision (more a vision by the mind than a vision by the eyes), which we would like to suggest to name mathematical intuition or mathematical imagination. For this last case, one can make a further distinction. There can be, on the one hand, an intuition that is essentially a direct (intrinsic) mental vision of, say, a mathematical objet such as a surface, a manifold, etc., or of a physical phenomenon, thereby we anticipate on the further mathematical exposition and phenomenological investigation of these same objects. On the other hand, we have an intuition which engages from the very outset some mathematical concepts. Generally, these two types of intuition operate at distinct levels of our consciousness and mathematical activity, but they can also interact.

Otherwise stated, the word "visualize" has two meanings: to make visible (form a visual image), and to imagine (form a mental image). According to J.J. Gibson, "A surface can be seen; a plane can only be visualized". (Gibson 1979). The term "surface" can have several meanings, whose two are very important for perception and for mathematics respectively. The common meaning, as meant by Gibson, refers to the perceived surfaces of objects such as mice, chairs and mountains (call them the real-world surfaces). The other is the abstract mathematical concept, an intrinsic two-dimensional manifold, which can be visualized only by the mathematical imagination. It is this second kind of visualiza- 
tion that is powerful in mathematics for it combines visual and non-visual information in a fruitful way.

For example, we can see real-world objects, surfaces and especially edges of these surfaces. We see (to a certain extent) colors; we organize what we see and, that is, we range (or classify) what we see according to some abstract schema; in other words, there are typically many objects in view, so we try to organize and group them and detect patterns of their arrangements. We detect small patterns with considerable repetition, called textures. Examples of textures are the distant pattern of trees as seen from a high altitude, the grain of wood on a tabletop or the nap of a piece of cloth. On all real world surfaces, we see colors and textures. We can detect motion and changes of appearance of objects, of groups and of textures. Motion plays an important role in the basic process of visual understanding of ordinary objects. She is important since it gives a continuous family of views. We have depth perception - the visual ability to judge distance from an object. This process is complex, with factors interrelated in ways that are still not well understood. Certainly an important aspect is binocular (stereoscopic) vision which allows mental triangulation. Additional important clues are also given by relative apparent motions of objects as we, or the objects move.

However, we cannot see the most the geometrical objects, but only visualize or imagine them in connection with the corresponding mathematical objects such as multidimensional space, ${ }^{5}$ invariants, connection, homeomorphism, homology, etc. As a first remark, we can say that

"three-dimensional manifolds in $\mathbf{R}^{4}$ are more difficult to visualize than two-dimensional surfaces in $\mathbf{R}^{4}$, since the ratio of necessary information to visible information is much smaller. To take a very important example in our-days developments of certain branches of pure mathematics, let's

$5 \quad$ N. Wiener has written: "Four-dimensional geometry certainly exists as a general form of [mental] deduction, but there is no reason whatever to assume the [physical] existence of four-dimensional space" (Wiener 1922). The word "four-dimensional geometry" refers to pure mathematical geometry, whereas in the expression "four-dimensional space" the word space can also refers to physical space. Indeed, it is 4-dimensional space-time that occurs in Einstein's general theory of relativity and not four-dimensional space. 
mention the topological knot theory and especially some of its fundamental facts, in order to show that a number of results related to this theory are completely counter-intuitive, although they need, to be grasped, a great deal of inventive intuition and mathematical imagination" (Roseman 1997, 444-445).

(i) In order to understand the mathematical signification of a knot we first need some basic knowledge about the concept of embedding, that is, the fact that, $X$ and $Y$ being topological spaces, an embedding of $X$ into $Y$ is a one-to-one bi-continuous map of $X$ into $Y$ - that is a homeomorphism of $X$ onto $f(X)$. Further, the fact that, if $X$ and $Y$ are smooth manifolds, an immersion of $X$ into $Y$ is a smooth function, $f$, from $X$ into $Y$ that is locally one-to-one but not necessarily globally one-to-one. Equivalently, we can define an immersion as a smooth map whose differential, $d f$, is non-singular. An immersed manifold is an image of an immersion. For example the limaçon, the graph in polar coordinates of $r=1 / 2+\cos \theta$, is an immersed circle in the plane. Another important example, the most familiar image of the Möbius band is a "circular band with a half-twist"call this subset $M_{1}$. This is an image of one embedding of this 2-dimensional manifold into ambient space $\mathbf{R}^{3}$. Recall the counter-intuitive fact that this manifold is non-orientable.

Let's now introduce the concept of isotopy, which is another fundamental concept of knot theory.

"From a topological point of view, it allows to define the equivalence relation of manifolds or subsets of $\mathbf{R}^{n}$. Two subsets $A_{1}$ and $A_{2}$ of $R^{n}$ are isotopic if there is a non-rigid motion in $R^{n}$ which takes $A_{1}$ to $A_{2}$. That is, there is an object, $A$, and continuous family of embeddings, $f_{t}: A \rightarrow R^{n}$ with $f_{1}(A)=A_{1}$ and $f_{2}(A)=A_{2}$, where $t \in I$, I being the unit interval. Such a map will be called an isotopy of $A$. Two subsets of $R^{n}$ are equivalent if there is an isotopy between them. Consider the Möbius band in $\mathrm{R}^{3}, M_{1}$, described above. Now let $M_{2}$ be a similar example: "circular band with three halftwists". Wee can show that $M_{1}$ and $M_{2}$ are not isotopic. To prove that, we have to consider that, if they were equiva- 
lent, then the corresponding boundary sets would be equivalent as circles in $\mathbf{R}^{3}$. But the boundary of $M_{1}$ is a trivial knot and the boundary of $M_{2}$ is a non-trivial knot called a trefoil knot" (Roseman 1997b, 68).

(ii) A knotted manifold is defined to be the image of an imbedding of a compact manifold into $\mathbf{R}^{n}$ for some $n$. The isotopy equivalence class of a knotted manifold will be called a knot. If we have one circle in $\mathbf{R}^{3}$ this knotted manifold is called a knotted circle. If we have several circles in $\mathbf{R}^{3}$ this knotted manifold is called a link. It is easy to create examples of knotted circles (or embedded circles) in $\mathbf{R}^{3}$. A knotted surface is a knotted 2-dimensional manifold, with empty boundary, in $\mathbf{R}^{4}$. This can be considered as a smooth sub-manifold or a piecewise-linear manifold-that is, a two-dimensional simplicial complex that approximates a smooth knotted manifold. Generally, surfaces in $\mathbf{R}^{4}$ (or even in $\mathbf{R}^{3}$ ) are difficult to describe and even more to visualize. Knotted circles are an important tool in the study of the topology of $\mathbf{R}^{3}$. It is central to the investigation of three-dimensional objects. It is also of interest to chemists and biologists since molecules (including DNA) have been found with a knotted structure. Furthermore, properties of these molecules seem to depend on the "knottedness" and its associated numerical and/or topological invariants like the linking number, the writhe number, and the twist number. In physics, remarkable connections have been found between quantum physics and knots, especially to knot diagrams and their representations (see Carter, 1995; Boi, 1994; 1995).

“To 'see' why knotted circles are key in topological understanding of three-dimensions, we need to know three basic mathematical facts about circles, which are completely counter-intuitive in the first sense ("empirical intuition") that we have attributed to the term "intuition". (a) There are no knotted circles in the plane. (b) There are many ways to knot a circle in $\mathbf{R}^{3}$. (c) There are no knotted circles in $\mathbf{R}^{4}$. In summary, $\mathbf{R}^{3}$ has the unique property that in it we can knot circles (1-dimensional spheres). Let's give some other profound and counter-intuitive mathematical properties and results concerning knotted surfaces in four-dimensions. 
(d) It is impossible to knot a sphere in $\mathbf{R}^{3}$. (e) There are non-trivial knotted spheres in $\mathbf{R}^{4}$. However, it is impossible to knot a sphere in $\mathbf{R}^{5}$. Thus $\mathbf{R}^{4}$ is the unique dimension for which spheres can knot. (f) An $n$-dimensional sphere can be non-trivially knotted in a piecewise linear manner in $\mathbf{R}^{m}$, if and only if $m-2=n »($ Roseman 1997a, 68).

(iii) The information about how surfaces knot in three-dimensional space, viewed mathematically, results in a structure called a knot diagram. In the mathematical study of knotted circles in three-dimensional space, the basic question is: given two knotted circles, are they equivalent? If knots are not equivalent, we generally distinguish them by means of topological or algebraic invariants, such as the knot group. There are many such invariants, but most are derived from diagram of a knot. On the other hand, if knots are equivalent, then we need to find an isotopy that shows this equivalence. Thus, symbolic/mental representation combined with physical gestures/operations is an important part of mathematics. The role of diagrams notably in geometry and topology is very important. In most of the situations, these diagrams are not used merely as "illustrations", but as complex symbolic operations of a geometric-algebraic nature. Furthermore, these diagrams enable to translate (or to reveal) some striking topological properties of the knot that is being studied. On the other hand, these topological properties, once known, may lead to discover new geometric and/or algebraic invariants of knots.

Then, a knot diagram is not simply an "image of a knot"-it is a powerful pictorial and conceptual representation of the knot, useful for further mathematical deepening of its properties. One can use the knot diagram directly to calculate many geometric and algebraic invariants of the knot. The knot group is one such invariant. If $M$ is a submanifold of $n$-dimensional space (or the $n$-dimensional sphere) the knot group of $M$ is defined to be the fundamental group of the complement of $M$ (see Boi, 1995; 2006; 2011). Operations on a given diagram can yield a family of related knots (the so-called knot skeins) and may be used to calculate a variety of knot invariants. As Kauffman so clearly pointed out in (Kauffman, 1988), knot diagrams and geometric manipulations of them 
provide powerful mathematical results-new topological invariants and new insights into previous ones.

\subsection{Back to Kant in the Light of Modern Mathematics and Physics}

Let's now go back to Kant. The idea (which Kant shares) that exists a sort of necessary correspondence between geometrical "axioms" (such as the three-dimensionality of our space and the essential uniqueness of the Euclidean geometry, which I already mentioned) and the evident intuitive content to which they refer, appear to be not well-founded and must therefore be replaced by a theory according to which mathematical objects are first and foremost objects of thought. And even if they may turn out to be abstract models allowing to explaining certain categories of phenomena, they not derive their raison d'être directly from the experience nor they do their existence to a priori and subjective laws.

We could say, therefore, that mathematical objects are "entities" of thought and forms or models of the phenomena at once. It is thus possible that a mathematical structure-for example, the invariants of a certain type of four-dimensional manifolds in our-days theoretical physics - can render (or can correspond to) the essential properties of physical phenomena. Returning to our example, this structure would be a group of internal symmetries describing the interactions between particles. On the other hand, most of the fundamental physical theories entail deep mathematical structures, which prove to be indispensable for explaining the behavior of phenomena, at the macroscopic aw well as at the microscopic level. General relativity, quantum field theory and gauge theory provides very good examples of such a situation. The major problem, for today's scientists and more generally for the theory of knowledge, lies in understanding the type of dynamical patterns linking geometrical and topological structures to the most streaking features of physical theories. Physical intuition and mathematical imagination can make a fundamental contribution to the attempts that are currently being made for developing theoretical models able to find sound mathematical structures corresponding to the physical behaviors of phenomena to be explained.

Let us mention two important examples.

1) In the eighteen it has been realized that gauge theory is essentially one branch of differential geometry which uses the new concepts of 
vector bundles and fiber spaces with connections, which are of purely mathematical character. Now these concepts have become central in the understanding of the connection between mathematical structures and physical theories, and they directly links geometry and physics; in fact one can say that the two are coextensive. Indeed, consider the mathematical concept of a space with a connection ${ }^{6}$ and its curvature (see Manin 1981).

"Let $f: M \rightarrow N$ be a map between spaces $M$ and $N$, where $M$, say, represents a model of space-time, and at each point $p$ of $M$ there is localized a physical system with the space of internal states $f^{-1}(p)$. A connection on geometrical object like a fiber space is a rule permitting the transport of the system along the curves in $M$. In other words, if we know part of the world-lines and the initial internal state of a system in $M$, then, thanks to the corresponding displacement determined by the connection, we can know the future states of the system. According to the recent physical theories, a gravitational field is a connection in the space of internal degrees of freedom of a gyroscope; the connection allows us to follow the evolution of the gyroscope in space-time. An electromagnetic field is also a connection in the space of internal degrees of freedom of a quantum electron; the connection allows us to follow the evolution of the electron in space-time. A Yang-Mills field is yet a connection, in the space of internal degrees of freedom of a quark. This geometrical image seems now to be the most universal mathematical model of an ideal universe with a small number of basic interactions. The state of matter in space-time, at each point and each moment, is described by a section of an appropriate fiber space $N \rightarrow M$. A field is described by a connection on this fiber space. Matter acts on the connection by imposing restrictions on its curvature, and the curvature, and the connection acts on matter by forcing it

6 Recall that Levi-Civita, E. Cartan and H. Weyl introduced the concept of connection in the early 1920's. 
to propagate by "parallel displacement" along world-lines. The famous equations of Einstein, Maxwell-Dirac and YangMills are exactly the embodiment of this idea. The geometrical concept of connection has thus become a fundamental ingredient of contemporary physic" (Boi 2004b, 1825-26).

So we realize that to each physical entity and their correlated physical-theoretical model corresponds a local or global structure of differential and algebraic geometry and topology. The so-called dictionary of Yang-Wu (1975) has very well illustrated this important fact. For example, field strength is identified with the curvature of the connection; the action integral is but a global measure of curvature. Certain topological-algebraic invariants in the theory of characteristic classes have been seen to be most appropriate to describe the charge of the particle in the sense of Yang-Mills. More generally, we can establish a direct correspondence from the concepts of gauge field theory to those of the differential and topological geometry of fiber spaces.

But how can we understand precisely the nature of such a correspondence? It should appear enough clear that it is neither of empirical nature, nor of logical character, nor of transcendental meaning at least such as Kant meant it. The a priori role of many mathematical invariants, as well as their constitutive role (that is to say their dynamical power of generating phenomena), is a very important scientific fact that requires a new philosophical analysis.

Here, we would like to suggest the idea that, fundamentally, physics is but geometry in act. This implies not only that geometry produces mathematical abstract concepts like manifolds, groups, curvature, connections, fiber spaces, etc., but also that it is, in a way, ontologically rooted in reality, because it participate somehow in the properties of physical entities and the qualities of phenomena.

"In fact, some principles of geometrical symmetry (or, equivalently, some groups) can be transformed into dynamical principles that are in turn responsible for certain changes in the phenomena. Thus, the concept of symmetry is not just abstract, and its mathematical properties have 
simultaneously an explanatory power and a capacity to generate a world of forces, interactions and energy, so that the mathematical understanding of this world cannot be separated of the understanding of reality itself. On one hand, one is increasingly led to believe that symmetry might determine at a deep level almost everything. Nevertheless, on the other hand, it is not unreasonable to look on topology, like symmetry, as some kind of underlying or unifying principle which helps us to understand complex (macroscopic and microscopic) physical phenomena" (Boi 2003, 216).

2) Theoretical physics is today faced with two key questions that are at the heart of the mathematical developments of quantum field theories, which include string theory, topological quantum field theory, non-commutative geometry and loop representation. The first question is: What we can mean by quantum geometry? Indeed, we have learned with general relativity that space-time geometry shares the same dynamical character of the electromagnetism field and the other physical fields; and we have learned with quantum theory that all dynamical objects exhibit quantum behavior: they can be in probabilistic superpositions of states and they manifest in quanta at small scale. If we trust the general validity of these two physical discoveries, we are led to conclude that space-time geometry too must exhibit quantum behavior: it may admit probabilistic superpositions and it may manifest itself in quanta at small scale. What is then space-time geometry with such features? What kind of mathematics can manifest it? What physical meaning can we give to it? We have a certain understanding of quantum field theory (QFT) on a fixed geometrical background; can we understand how to describe a world in which the background geometry, space-time itself, is quantum mechanical?

The second question is how to build a diffeomorphism invariant QFT. This is, in a sense, the mathematical translation of the first question.

"To illustrate the physical need of a diffeomorphism invariant quantum field theory, consider the following observations. Space-time geometry is dynamical, and thus rather similar to physical fields-indeed, space-time geometry is the gravitational field; but we can also turn this observation 
around and notice that the dynamics of the gravitational field is profoundly peculiar-indeed, the gravitational field is space-time geometry. In fact, this dynamics cannot be captured, as other physical fields, by techniques that rely on the existence of a fixed background space-time. More precisely, as soon as we include the gravitational dynamics into any field theory, we are forced to an interpretative as well as a mathematical jump: Since the background geometry is itself a dynamical object, spatial and temporal localization cannot be defined with respect to non-dynamical background structures. Localization can only be defined with respect to dynamical entities. It seems that this "relational" view of space-time is the basic physical idea whereby general relativity has contributed to our understanding of the natural world. In the mathematical formulation of the theory, this physical idea is realized by a gauge principle, which is invariant under diffeomorphism. To fully incorporate diffeomorphism invariance into quantum field theory, therefore, seems to require that we abandon local quantum field theory, in which physical operators are labeled by regions of a metric manifold, in favor of a diffeomorphism invariant quantum field theory, in which physical operators are diffeomorphism invariant objects. Therefore, the definition and the construction of a nontrivial diffeomorphism invariant quantum field theory is at present one of the most fascinating problem, and in our opinion it is very important in order to be able to clarify the relationship between physical intuition and mathematical conceptualization" (Rovelli 1995, 6530-31).

\section{Merits and Limits of the Kantian Notion of "Synthetic a priori"}

From the previous discussion, it should now be fairly evident that Kant's conception of intuition is somewhat problematic, and that even when we try to replace the neo-empiricist's reductive and fundamentally incorrect interpretation, with a more balanced, revised interpretation of 
these same difficulties (which we have sought to enumerate briefly here), the inadequacy and ambiguities of such conception are still plain to see. The main problem lies at the heart of the Kantian system and Kant's way of reasoning which prevent him from coherently develop his system. As Jules Vuillemin wrote, the main difficulty with Kantian lies in his notion of intuition. He goes on to say that

"Contrary to what asserted by the axioms (in the Transcendental Aesthetic), according to which "the representation of space is intuitive" [or similarly, "geometry proceeds synthetically, that is to say, by sensual intuition"], intuition does not necessarily come into consideration in geometrical proofs, nor does it correspond to the intrinsic meaning of the axioms...(Vuillemin 1994, 351).

The other fundamental question that Kant considered, and which in his reasoning is inseparable from that concerning the role of intuition, is to know if and how synthetic a priori judgments are possible, and more particularly, how they are possible in mathematics and theoretical physics. We know the answer putted forward by neo-empiricism and logicism, which found the question herself to be devoid of sense. Firstly, in mathematics, because all mathematical concepts can be reduced to purely logical notions (this is notably the point of view of Frege and Russell), thus rendering all inner intuitions and synthetic judgments unnecessary. Secondly, in physics, because of the empirical nature of its concepts, which contradict the supposed a priori nature of the notions of space and time. However, it seems rather clear that this interpretation of Kant, and this conception of modern mathematics and physics are essentially wrong. For more convincing evidence of this, we should look at the development of mathematical logic with Zermelo-Frankel's and Gödel's theorems, as well as at the developments occurred in the second half of last century in the fundamental domains of mathematics such as differential geometry, algebraic and differential topology and the theory of dynamical systems, etc. The same may be said for developments in theoretical physics, notably with general relativity, gauge theory and topological quantum field theories. 
The question raised by Kant is therefore relevant, but for it to be wellfounded, we must improve the conception of what is meant by a priori and substantially modify the status of intuition. We have to realize that the possibility of synthetic a priori judgments in mathematics does not necessarily require that knowledge' process be submitted to the effects of inner subjectivity, since intuition or imaginative thought coupled with concepts could also serves to that purpose.

This was the solution proposed by Poincaré in his analysis of the formation of the notion of geometric space and the role of the concept of group in the constitution of geometry. He showed that the notion of space couldn't be formulated on the basis of simple spatial sensations, since the latter do not share the same properties which characterize abstract geometrical objects, notably their properties of homogeneity, isotropy and infinitude. Moreover, he stressed the fact that the concept of group of transformations (Poincaré refers to continuous Lie groups), which is of fundamental importance in the development of geometry, is a fundamental concept of our understanding of space, which enables us to reconstruct the whole mathematical structure of a certain type of geometry. For example, the group of Euclidean movements is all what we need in order to give a complete definition of Euclidean geometry. Moreover, geometric space-whether this be Euclidean or non-Euclidean-, instead of being a pure form of our sensual intuition, or even a form of transcendental subjectivity, is rather a mathematical object which is obtained from the action of a certain group on some "primitive" space, that is to say, a space still lacking of all mathematical structures it might bear: metric, differentiable, algebraic, and topological. For Poincaré, nonetheless, the concept of group is fundamental for our understanding of the inner structures of physics, and it is also a profound tool of our intuition of space, in the mathematical and creative sense of the word. However, at the same time, there is a synthetic meaning to be found in the concept of group, the expression of which concerns less the way in which it fits into the mathematical reasoning, or the internal development of its formal contents, than to the fact that it make possible: (a) To generate new spaces, and hence new mathematical beings which can be defined within these spaces; (b) To deduce, by means of either geometrical symmetries (e.g. spatial-temporal symmetries as is 
the case for general relativity), or internal symmetries related to the phase in quantum mechanics (rotations, spins, non-commutative operations, duality), which have been found to be associated to the new spaces, certain fundamental and dynamical properties of physical phenomena. This deduction is neither empirical nor logical in nature; it is rather, in some way, constitutive of phenomena. This fact considerably widens our notions of "synthetics a priori" and intuition in physics and mathematics.

We think that it is still conceivable to speak about the transcendental character of mathematical physical theories, provided that we abandon the two following Kantian axioms: (1) Space and time no longer belong to the realm of specific or autonomous intuition, (2) The synthesis of the unity of apperception (Aperzeption) is not necessarily based on an a priori form; instead we have the geometrical structures and the theoretical models that allows for some objectivity of physics and of natural phenomena.

In the light of the previous considerations and for some important reasons tied up with some developments in mathematics and physics in the 19 th and 20th centuries, we suggest reinterpreting Kant's transcendental concepts and appraising their value in a limited and "local" manner. We should firstly consider that in Kant's time, Euclidean geometry and Newtonian physics occupied a dominant position. Actually Kant built his philosophical system from these two theories, considered by him models of scientific rationality and objective knowledge. For Kant, Euclidean geometry was to be taken as an unquestionable fact, for two reasons: (1) Firstly, because our spatial intuition unfolds within the Euclidean geometric scheme, (2) Secondly, because our usual space is physically Euclidean, and can, therefore, be described by Newtonian physics.

We should be very careful about these two postulates, which Kant considered as self-evident; if they are "true" they are only partially and/ or locally true. It seem to us that, if, on the one hand, some general ideas-most notably the ones which asserts that "geometry is a science which determines the properties of space in a synthetic, although a priori manner (...) and that only this assumption allows to understand the possibility of geometry as a synthetic a priori knowledge"-bear some validity and importance for the current research in mathematics and 
physics, on the other hand, the specific contents of these ideas appear somehow obscure and wrong.

A few more comments on this subject are in order. The axiomatic movement, and in particular the work of Hilbert and others on the foundations of geometry and arithmetic, have helped to show the importance of the distinction between mathematical space and physical space. The laws governing the former are considered to be the necessary consequence of a small number of axioms to which we have from the outset ascribed the status of free logical statements. And any theoretical domain, belonging to mathematics or to mathematical physics is considered as a formal system of abstract objects, which are mathematically well-defined but physically undetermined, and which, as constitutive elements, will be part of the theoretical re-construction of the real world. Moreover, what is more significant for us, and what $\mathrm{H}$. Weyl particularly stressed, is that the philosophical interpretations, after Kant's conception, of the significance and pertinence of the synthetic a priori judgments in science, have, over time, evolved in two different directions. On the one hand, there are non-empirical laws of "essences" (Wesengesetze) that describe the manner in which the levels and contents of consciousness develop in relation to one another, although they do not involve factual propositions. Husserl developed this point of view as part of his phenomenological investigation, where the notions of a priori and intuition are more fruitful than they have ever been in the Kantian system. On the other hand, there are principles of a conceptual construction whatever it can be (in mathematics or theoretical physics), which, according to the Poincaré's point of view, rests on some theoretical assumptions or (semantic) conventions.

Within this framework, we would like to add few remarks in order to better elucidate the phenomenological point of view of Husserl and Weyl. For the comprehension of Husserl's conception one central aspect concerns the meaning that we must ascribe to what he called the "identity of thing" and its essential property, namely the continuity (see particularly Thing and Space...). It is a matter of distinguishing between phenomenological spatial continuity and mathematical spatial continuity (which is ideally hypothesized and scientifically determined), and also between the factual identity (sachlich Identität) and its objective identity. Husserl had 
made the distinction, with regard to the characterization of the identity of the empirical things, between its "actual changes" and its "possible changes".

This distinction implies two profoundly different conceptions of continuity. According to one, continuity is of phenomenal nature and serves to determine the totality of the actual changes of the appearance of an object; these moments are held together in the consciousness of immediately immanent unity and occur contemporaneously with the act(s) of perception. According to the other, continuity, wherein the infinite is permitted to intervene into the kind of possible perceptions, cannot be enclosed within the limits of an immediately given and ever-similar unity. The invariant unity of the object could eventually be given, but in an altogether different manner and namely by an a priori mathematically defined process. This difference, which Husserl points out on more than one occasion, must be understood in relation to his discussion of the concept of space as a continuum of real phenomena- "filled" in part by the ostensive and physical properties of body-surfaces present herein, and partly by the sense qualities (qualia) of these same body-surfacesand space as a continuum defined in terms of formal category: the "form" of the Euclidean manifold or of any $n$-dimensional manifold, be it Euclidean or not. This last type of continuity is in fact inseparable from the mathematical rigorous concept of infinity.

Relying partly open this Husserl's view, Weyl has proposed the twofold following thesis (see Das Kontinuum, 1918). In the first place, he asserts that it is impossible to found the intuitive (phenomenal) continuum on the mathematical continuum, and that, in turn, mathematics is not based on the intuitive continuum, but rather on the category of natural numbers. In the second place, he maintains that the two kinds of continua are essentially different with respect to their ontological properties, in the sense that it seems problematic, for example, to build a pure theory of time starting with the concept of numbers. In fact, this leads to the idea that an autonomous theory of the continuum is conceivable independently from number theory or an arithmetical background. The fact that the only scientifically viable theory of continuum is one that rests on the mathematical concept of real numbers, doesn't exclude: (i) First, that one can mathematically distinguish and construct several possi- 
ble models of the continuum that, though not necessarily isomorphic, can nevertheless be all ideally meaningful and useful for the theoretical explanation of the physical world, (ii) Second, the possibility of developing a truly philosophy of continuity which, instead of exclusively resting upon the concept of natural numbers, hence on the discreteness of numbers, should try rather to rely on some purely continuous topological properties, such as homeomorphism of smooth surfaces and manifolds, embedding and immersion of these same surfaces and manifolds, and the fundamental equivalence relation of isotopy. ${ }^{7}$

For example, consider again two objects $A_{1}$ and $A_{2}$ (or two surfaces $S_{1}$ and $S_{2}$ ). To prove that $A_{1}$ and $A_{2}$ are isotopic, by a continuous family of embeddings (i.e., a smooth map or motion in $\mathbf{R}^{n}$ which takes $A_{1}$ to $A_{2}$ ), one has to show qualitatively that their corresponding boundary sets or subspaces are equivalent circles (in the case of two-dimensional manifolds) in Euclidean space $\mathbf{R}^{3}$. At any rate, we think that an enlargement of the mathematical concept of continuity be possible, provided however that we discard the set-theoretic conception of the existence of an unique and absolute mathematical ontology, ${ }^{8}$ i.e. the platonic world of numbers

$7 \quad$ For an interesting attempt in this direction, see Thom 1992.

8 See on this subject the very helpful comments made by G. Longo in 1999. Let me quote him in extenso: "In this article we have underlined, initially, the non-uniqueness of the intuition of the continuum. Then we have developed an analysis that emphasized three levels: the intuition one, the construction principles one, and the proof principles one. On the first level, the richness of the world and of points of view from which to observe it, compatible points of view, non isolated, but built from a dialogue with evolution and history, suggest a plurality of intuitive approaches and ground mathematics in our relation to the world. In part we find these points of view in the different mathematical constructions of the continuum, which constitute the second level. These constructions enrich and modify the original intuitions (...). But thanks to Logic there is a third level, where the analysis of the proof (...) plays an essential role. Clearly, the incompleteness results lay in between the second and the third level, as a precise form of indetermination of mathematical constructions by formal theories. (...) In the case of the continuum, the mathematical objectivity is also in the richness of interactions of three levels we mentioned: intuition, Mathematics, Logic. This interaction is not a vicious circle, but a virtuous one, extraordinary example of the dynamicity of our forms of knowledge" (p. 21-22). And still more significantly he go on by stating: "We cannot 'found' mathematics (...) over a mathematical discipline, a logical-mathematical system also made up of mathematical 'rules of the game' (as Wittgenstein says). There cannot be an internal foundation, purely formal and mathematical, of Mathematics: the incompleteness theorems are not accidents, they underline the gap between the mathematical principles of proof (...) and the rigorous practice of mathematical constructions" (Ibid.). 
and sets, and we recognize besides: (i) Firstly, the possibility of several modes and principles of construction of mathematical beings or objects; in other words, the likely existence of several mathematical ontologies, (ii) And secondly, the possibility that it can exist a connection between different "real" ontologies (for example, physical, biological) and imaginary (such as literary, artistic) ontologies and the mathematical ones.

Now let's return to Poincaré. The conventional principles he introduced are not arbitrary or devoid of semantic significance, they are, in some sense, constitutive for the science of space; yet in a different manner to that Kant or even Husserl proposed. Firstly, it is a matter of a formal constitution, in the "qualitative" (structural) and not logical sense of the word. We can in fact show that the mathematical conventions Poincare spoke about are essentially non-syntactic and non-pragmatic. According to $\mathrm{H}$. Weyl, the properties we ascribe to physical space are both a priori and synthetic, and entail a certain type of objective meaning, which don't necessarily require that we assign them a subjective origin, as Kant did. On this point, Weyl emphasizes the fact that, according to Riemann's and Einstein's point of view, one can oppose the Euclidean-Pythagorean nature of the metric, the only element which is given in an "absolute" manner and which does not participate in the manifest indetermination of what occupies a variable place in the spatial-time continuous, to the reciprocal orientation of the metric at different points, that is to say, the quantitative and qualitative evolution of the metric field (i.e. the gravitational field in general theory of relativity), the latter being contingent and dependent upon the presence and distribution of matter.

Thus, the general relativity theory does not, in this sense, completely deny the existence of an a priori given entity inherent to the structure of the extensive medium of the external world; all that it maintains is that the frontier between a priori and a posteriori has, in this case as well in the more general scientific and epistemological context, shifted. For Riemann, in addition to the a priori features of the universe, and besides the basic nature of the Euclidian-Pythagorean metric, there is the topological connectedness, which is primitively given, and notably the number of its dimensions being 4-this is obviously the space-time dimension of general relativity. Moreover, the quantitative and qualitative evolution of the metric field obeys precise natural laws, particularly Einstein's law of 
gravity, which is similar to Maxwell's electromagnetic field. Thus, we can make, as suggested by Weyl, another distinction within the a posteriori category, between what seems to be necessary, in terms of natural laws, and what remains free and contingent, whilst obeying exact principles.

\section{The Concept of Space and Its Constitutive Levels: From Kant to Riemann and Weyl}

Weyl repeatedly stressed that the understanding of the complex relationships between geometric, physical and perceptual space, is one of the most fundamental scientific and philosophical issues of our time. These three kinds of space form the constitutive levels (konstitutive Stufen) of the general concept of space. Furthermore, Weyl proved two important insights. He has shown that geometric space (his prototype $\mathbf{R}^{3}$, or $\mathbf{R}^{n}$, or even any open neighborhood of $\mathbf{R}^{n}$ endowed with its intrinsic geometry) is different from the surrounding Euclidean space $E$ (das extensive Medium der Aussenwelt), essentially because it is a mathematical model of the concept of space. He has also shown that physical space is a type of actual existence (or a deformation) of the geometric space; and that we can, in fact, conceive several possible deformations. To this end we think, along with Poincaré, that the representative or perceptual space too, is a deformed image of the geometric space that obeys primarily certain laws of optics, perspective, and physiology.

Contrarily to Kant and in agreement with Riemann, Weyl maintains that it isn't the geometric space as such, and especially not its axioms, that are given a priori. It is rather the space of our immediate experience or the surrounding living space that form a sort of amorphous topological continuum. This continuum is a primitive datum of our intuition, and we actually discover its qualitative nature as soon as we relate it to the perception of objects in the external world. Geometric space, on the other hand, can be obtained, according to Weyl, by a process which consists of supplying that amorphous continuum with some essential mathematical structures that mutually implicate each other: metrical, differentiable, algebraic, topological, and so on.

It was Riemann who first developed an entirely new conception of mathematical space on which differential geometry and topology, as well 
as a large part of modern physics, rests. But given its much more general range, especially for the understanding of the connection between the mathematical concept of manifold and physical space, it seems useful to review briefly some key ideas of the German mathematician.

First, the mathematical concept of manifold (Mannigfaltigkeit) seems to be very fundamental. A manifold can be intrinsically defined as any (small) part of $n$-dimensional space $\mathbf{R}^{n}$ endowed with a proper geometric (both metrical and topological) structure. Any surface can then be conceived as a two-dimensional space equipped with a set of fundamental geometric properties, independently of its specific relationship to the surrounding Euclidean space, hence as a genuine mathematical object. Similarly, a line will be considered as a one-dimensional manifold, and space as a three-dimensional manifold. We can, therefore, safely extend our conception of manifolds to higher-number and even infinite dimensions and treat them as autonomous mathematical beings. But not all surfaces or manifolds exhibit the same mathematical properties. The study of these properties has led to a new fundamental distinction between metric (local) and topological (global) properties. As a consequence, it became clear for the first time that manifolds that are equivalent in terms of their (local) metric properties (e.g. a plane and a cylinder) can be altogether different with regard to their (overall) topological properties. Accordingly, these mathematical objects can be classified on the basis of their intrinsic properties and their respective structures. This led to the important idea that two manifolds, which are metrically or topologically equivalent, belong to what would henceforth be known as a same spatial form. The surfaces or $n$-dimensional manifolds that can be continually deformed one into the other, while their metric relations remain invariant, are thus called isometric. Those, on the other hand, that can be deformed through a bi-continuous global deformation are called homeomorphic (topologically equivalent).

The previous implicitly embraces three other fundamental ideas introduced by Riemann and developed notably by Weyl. It is useful to highlight their importance here, particularly because this allow for a better understanding of the spatial intuition and of our phenomenological space. (i) All manifolds can be endowed with several metric structures; from this point of view, Euclidean geometry may be treated as one among other 
possible structures. (ii) Manifolds can be distinguished with respect to whether they are continuous or discrete; the first are endowed with a metric (differential) structure; the second possess an essentially algebraic structure. One important consequence of such a distinction is that we can think of different metric forms and differentiable structures for the same topological space. (iii) All manifolds can support different kinds of structures that are at once metric, differentiable, topological, algebraic, etc., and this is what essentially makes their mathematical interest. (iv) There is an important distinction to be done, between the boundlessness (Unbegrenzheit) and the infinite (Unendlichkeit). While the first is a topological property, the second is a metric one. In other words, we can very well think of a manifold as being finite and boundless at the same time. Let's take the example of the sphere $\mathbf{S}^{2}$. One can go around it an unlimited number of times, but since topologically the sphere is a closed space and all its points are mutually identical, we can only trace finite movements because we can't leave the space it encloses without breaking its boundary and, no matter where we start our movement we will return exactly to our initial point. In short, the sphere is a model of a finite and unbounded space.

To conclude, let's make the following three remarks.

(i) The fact that space is an unbounded three-dimensional manifold is an assumption which is required in order to develop our conception of the external world, for it is exactly this assumption that enables us to complete our perception of the possible positions of objects and further to construct a full spatial characterization of these objects. From this point of view, the three-dimensionality of space isn't actually an $a$ priori condition of our sensual perception or subjective knowledge. (ii) However, mathematically as well as physically, space can be at the same time metrically infinite and topologically finite (closed); and also it can have more than three dimensions and even, in fact, an infinite number of dimensions..$^{9}$ (iii) The perceptual space is not necessarily Euclidean. In fact, for example, the idea that the laws characterizing visual space

$9 \quad$ For an interesting and detailed discussion of this topic see, for example, R. Penrose, "Structure of space-time", in Battelle Rencontres, C.M. DeWitt \& J.A. Wheeler editors, W.A. Benjamin, New York, 1968, 121-235. 
were the same as those pertaining to a finite bi-dimensional (i.e. spherical) space has already been formulated towards the end of the 19th century. The Scottish philosopher Thomas Reid, among others, while examining the geometric properties of the visual field that he believed to be spherical, recognized important analogies between the geometry of the sphere and the geometric structure of visual space. Accordingly, he was led to make the distinction between tactile geometry, which is Euclidean, and the geometry of the visual field which follows other laws than the Euclidean ones, specifically spherical. Indeed, recent theories of perception (see Suppes (1977); Gordon (1997); Boi (2004)) confirm the fact that the geometrical structures of perceptual space are more complex and essentially different from those which characterize our Euclidean surrounding space.

\section{References}

Angel, R.B. (1974), "The Geometry of Visibles", Noûs, 8 (2), 87-1 17.

Atiyah, M. (1979), Geometry of Yang-Mills Fields, Lezioni Fermiane, Accademia Nazionale dei Lincei, Scuola Normale Superiore, Pisa.

- (1987), "On the work of Simon Donaldson", pp. 3-6 in Proceedings of the International Congress of Mathematicians (Berkeley, CA, 3-11 August 1986), vol. 2. Edited by A. Gleason. American Mathematical Society, providence, RI.

- (1988), "Topological quantum field theories", Publications Mathématiques de I'IHÉS, 68(1), 175-186.

- (1988), "New invariants of three and four manifolds", in The Mathematical Heritage of Hermann Weyl, Proc. Symp. Pure Math., 48, Amer. Math. Soc., 285-299.

- (1990), The geometry and physics of knots, Lezioni Lincee, Cambridge University Press.

- (1997), "Geometry and physics: Where are we going?", in Andersen et al. (eds.), Geometry and physics, Proceedings, Lecture Notes in Pure and Applied Mathematics, 184, Dekker, New York, 1-7.

- Atiyah, M. et al. (1990), "Responses to 'Theoretical mathematics': toward a cultural synthesis of mathematics and theoretical physics", 
edited by A. Jaffe and F. Quinn, Bulletin of the American Mathematical Society, 30 (2).

Baez, J. and J.P. Muniain (1994), Gauge Theories, Knot and Gravity, Series on Knots and Everything - Vol. 4, World Scientific, Singapore.

Becker, O. (1930), "Die apriorische Struktur des Anschauungsraum", Philosophischer Anzeiger, 4, 129-162.

Becker, O. (1973), Mathematische Existenz. Untersuchungen zur Logik und Ontologie mathematischer Phänomene, Max Niemeyer Verlag, Tübingen.

Benacerraf, P. and H. Putnam (1987), Philosophy of Mathematics. Selected readings, Cambridge University Press, New York.

Bennequin, D. (2001), "Invariants contemporains", in Panoramas et Synthèses, Société Mathématique de France, Paris, 11, 131-159.

Boi, L. (1989), "Objectivation et idéalisation, ou des rapports entre géométrie et physique", Fundamenta Scientiae, 10 (1), 85-114.

- (1992), "The 'Revolution' in the Geometrical Vision of Space in the Nineteenth Century, and the Hermeneutical Epistemology of Mathematics", in Revolutions in Mathematics, D. Gillies (ed.), Oxford University Press, Oxford, 183-208.

- (1993), "Mannigfaltigkeit und Gruppenbegriff. Zu den Veränderungen der Geometrie im 19. Jahrhundert", Mathematische Semesterberichte, 25, 10-35.

- (1995a), Le problème mathématique de l'espace. Une quête de l'intelligible, préface de R. Thom, Springer-Verlag, Heidelberg/Berlin.

- (1995b), Boi, L., "Conception 'dynamique’ en géométrie, idéalisation et rôle de l'intuition", Theoria, 10(22), 145-161.

- (1996), "La conception qualitative des mathématiques et le statut épistémologique du concept de groupe", in Henri Poincaré: Science et Philosophie, G. Heinzmann et al. (eds.), A. Blanchard/Akademie Verlag, Paris/Berlin, 425-449.

- (1997), Boi, L., "La Géométrie: clef du reel? Pensée de l'espace et philosophie des mathématique”, Philosophiques, 24 (2), 389-430. 
- (2001), "Sur la nature des objets mathématiques et les relations entre géométrie et physique", in De la science à la philosophie. Hommage à Jean Largeault, M. Espinoza (ed.), L'Harmattan, Paris, 197-246.

- (2003), "Philosophy of space-time", in Cambridge History of Philosophy 1870-1945, T. Baldwin (ed.), Cambridge University Press, Cambridge, 207-218.

- (2004a), "Theories of space-time in modern physics", Synthese, 139 (3), 429-489.

- (2004b), "Geometrical and topological foundations of theoretical physics: from gauge theories to string program", International Journal of Mathematics and Mathematical Sciences, 34, 1777-1836.

- (2004c), "Questions regarding Husserlian geometry and phenomenology. A study of the concept of manifold and spatial perception", Husserl Studies, 20 (3), 207-267.

- (2006a), "Nouvelles dimensions mathématiques et épistémologiques du concept d'espace en physique relativiste et quantique", in L'espace physique, entre mathématique et philosophie, M. Lachièze-Rey (ed.), EDP Sciences, Paris, 101-133.

- (2006b), "The Aleph of Space. On some extensions of geometrical and topological concepts in the twentieth-century mathematics: from surfaces and manifolds to knots and links", in What is Geometry?, G. Sica (ed.), Polimetrica International Scientific Publisher, Milan, 79-152.

- (2006c), "Mathematical Knot Theory", in Encyclopedia of Mathematical Physics, J.-P. Françoise, G. Naber, T.S. Tsun (eds.), Elsevier, Oxford, 399-406.

- (2009a), "Geometria e dinamica dello spazio-tempo nelle teorie fisiche recenti. Su alcuni problemi concettuali della fisica contemporanea", Giornale di Fisica, 50 (1), 1-10.

- (2009b), "Ideas of geometrization, geometric invariants of low-dimensional manifolds, and topological quantum field theories", International Journal of Geometric Methods in Modern Physics, 6 (5), 701-757.

- (2009c), "Images et diagramme des objets et de leurs transformations dans l'espace", Visibles, 5, 45-78. 
- (2011 a), The Quantum Vacuum. A Scientific and Philosophical Concept, from Electrodynamics to String Theory, and the Geometry of the Microscopic World, The Johns Hopkins University Press, Baltimore.

- (2011b), Morphologie de l'invisible. Transformations d'objets, formes de l'espace, singularités phénoménales et pensée diagrammatique, Presses Universitaires de Limoges.

- (2012a), "Fondamenti geometrici e problemi filosofici dello spaziotempo. Dalla relatività generale alla teoria delle supercorde", Isonomia - Rivista di Filosofia, 1, 1-37.

- (2016), "Imagination and Visualization of Geometrical and Topological Forms in Space. On Some Formal, Philosophical and Pictorial Aspects of Mathematics", in Philosophy of Science in the $21^{\text {st }}$ Century-Challenges and Tasks, O. Pombo and G. Santos (eds.), Documenta 9, CFCUL Lisbon, 163-221.

Bombieri, E. (2000), "Problems of the Millenium: The Riemann Hypothesis", CLAY.

Brouwer, L.E.J. (1912), "Intuitionism and Formalism", Bulletin of the American Mathematical Society, 20, 81-96.

Brouwer, L.E.J. (1975), Collected Works, Vol. 1: Philosophy and Foundations of Mathematics, North-Holland, Amsterdam.

Carter, J.S. (1995), How Surfaces Intersect in Space. An Introduction to Topology, Series on Knots and Everything, Vol. 2., World Scientific, Singapore.

Cassirer, E. (1910), Substanzbegriff und Funktionbegriff, Springer, Berlin.

Cavaillès, J. (1962), Philosophie mathématique, Hermann, Paris.

Châtelet, G. (1988), "Intuition géométrique et intuition physique", CISM, Courses and Lectures, No. 305, Springer-Verlag, Berlin Heidelberg, 100-114.

Clifford, W.K. (1879), "The Philosophy of Pure Sciences", in: Lectures and Essays, Vol. I, Macmillan, London, 254-340.

Conrey, B. (2003), "The Riemann Hypothesis", Notices of AMS, March, 341-353. 
Coxeter, H.S.M. (1998), Non-Euclidean Geometry (sixth ed.), The Mathematical Association of America.

De Jong, W.R. (1997), "Kant's Theory of Geometrical Reasoning and the Analytic-Synthetic Distinction. On Hintikka's Interpretation of Kant's Philosophy of Mathematics", Studies in History and Philosophy of Science, 28 (1), 141-166.

Desanti, J.-T. (1983), Les idéalités mathématiques, Seuil, Paris.

Donaldson, S.K. (1983), "An application of gauge theory to the topology of 4-manifolds", Journal of Differential Geometry, 18, 279-315.

Eilan, N., McCarthy, R. and B. Brewer (Eds.) (1993), Spatial Representation. Problems in Philosophy and Psychology, Oxford University Press, Oxford.

Feist, R. (2004), Husserl and the Sciences, University of Ottawa Press.

Feist, R. (2002), "Weyl's Appropriation of Husserl's and Poincaré's Thought", Synthese, 132 (3), 273-301.

Freedman M. and Quinn F. (1990), Topology of 4-Manifolds, Princeton University Press, Princeton.

Friedman, M. (2013), Kant's Construction of Nature: A Reading of the "Metaphysical Foundations of Natural Sciences", Cambridge University Press.

Friedman, M. (1992), Kant and the Exact Sciences, Harvard University Press.

Friedman, M. (1985), "Kant's theory of geometry", The Philosophical Review, Vol. XCIV, No. 4, 455-506.

Heijenoort, J. van, (ed.) (1967), From Frege to Gödel, Harvard University Press, Cambridge, MA.

Gibson, J.J. (1979), The Ecological Approach to Visual Perception, Houghton Mifflin, Boston.

Gödel, K. (1986), Collected Works, Vol. 1, edited by S. Feferman et al., Oxford University Press, Oxford.

Gonseth, F. (1974), Les Mathématiques et la Réalité, A. Blanchard, Paris. 
Goodman, N.D. (1979, "Mathematics as an objective science", American Mathematical Monthly, 86 (7), 540-551.

Gromov, M. (2000), "Spaces and Questions", in: Visions in Mathematics. GAFA 2000, special volume, N. Alon et al. (Eds.), Birkhäuser, Basel, 118-161.

Gordon, I.E. (1997), Theories of Visual Perception, John Wiley \& Sons, Chichester.

Hadamard, J. (1945), The psychology of invention in the mathematical field, Princeton University Press, Princeton.

Helmholtz, H. (1997), Epistemological Writings, edited by R.S. Cohen and Y. Elkana, Boston Studies in the Philosophy of Science, Vol. 37, D. Reidel, Dordrecht.

Hilbert, D. (1899), Die Grundlagen der Geometrie, Teubner, Leipzig.

Hilbert D., Cohn-Vossen S. (1932), Anschauliche Geometrie, Springer, Berlin.

Hintikka, J. (1996), La philosophie des mathématiques chez Kant, PUF, Paris.

Husserl, E. (1921), Logische Untersuchungen VI, zweiter Band: Elemente einer Phänomenologischen Aufklärung der Erkenntnis, Max Niemeyer, Halle.

- (1973), Ding und Raum, Vorlesungen 1907, edited by U. Claesges, Martinus Nijhoff, The Hague.

- (1983), Husserliana - Collected Papers, Vol. XXI: Studien zur Arithmetik und Geometrie, Edited by I. Strohmeyer, Martinus Nijhoff Publishers, The Hague.

Kant, I., Kritik der reinen Vernunft (1781-1787), new ed.: Meiner, Hambourg, 1990.

- (1786), Metaphysische Anfangsgründe der Naturwissenschaft, J.F. Hartknoch, Riga.

- (1980), Opus Postumum, translated and presented by F. Marty, PUF, Paris.

Kauffman, L. (1988), "New invariants in knot theory", American Mathematical Monthly, 95, 195-242. 
- (1987), On Knots, Princeton University Press, Princeton.

Kitcher, P. (1984), The nature of mathematical knowledge, Oxford University Press, New York.

- (1988), "Mathematical Progress", Revue Internationale de Philosophie, special issue on "Philosophy of Mathematics", P. Kitcher (ed.), 42 (167), 518-540.

Klein, F. (1979), Vorlesungen über die Entwicklung der Mathematik im 19. Jahrhundert (first ed., 1927), Springer-Verlag, Berlin Heidelberg.

Kreisel, G. (1965), "Mathematical Logic", in Lectures in Modern Mathematics, Wiley, New York, 95-195.

Kronheimer P. and Mrowka T. (1994), "Recurrence relations and asymptotic for four-manifolds", Bull. Amer. Math. Soc., 30, 21 5-221.

Lakatos, I. (1978), Mathematics, science and epistemology, Philosophical Papers, Vol. 2, Cambridge University Press, Cambridge.

Largeault, J. (1990), "Formalisme et intuitionnisme en philosophie des mathématiques", Revue philosophique, 3 (1990), 521-546.

- (1993), Intuition et intuitionnisme, Vrin, Paris.

Lautman, A. (1937), Essai sur les notions de structure et d'existence en mathématiques, Hermann, Paris.

Lawson, H.B.Jr. (1985), The Theory of Gauge Fields in Four Dimensions, Conference Board of the Mathematical Sciences, Regional Conference Series in Mathematics, No. 58, American Mathematical Society, Providence.

Mac Lane, S. (1980), "The genesis of mathematical structures, as exemplified in the work of Charles Ehresmann", Cahiers de topologie et géométrie différentielle, 21 (4), 353-365.

- (1986), Mathematics, Form and Function, Springer-Verlag, New York.

Maddy, P. (1980), "Perception and Mathematical Intuition", The Philosophical Review, 89 (2), 163-196.

- (1997), Naturalism in Mathematics, Clarendon Press, Oxford, 1997.

Manin, Yu I. (1981), Mathematics and Physics, Birkhäuser, Basel.

Milnor, J.W. (1965), Lectures on the h-cobordism theorem, Princeton University Press, Princeton. 
Montonen, C., and D. Olive (1997), "Magnetic monopoles as gauge particles?", Phys. Lett., 72B, 117-120.

Mumford, D., Series, C. and D. Wright (2002), Indra's Pearls. The Vision of Felix Klein, Cambridge University Press.

Murasugi, K. (1996), Knot theory and its applications, Birkhäuser, Boston.

Neumann, J. von (1961), "The Mathematician. 'The work of the mind'”, in Collected Works, Vol. I, Pergamon Press, London, 1-9.

Peirce, Ch. (1976), The New Elements of Mathematics, Vol. IV: Mathematical Philosophy, edited by C. Eiselle, Mouton \& Co. Publishers, The Hague.

Penrose, R. (1989), The Emperor's New Mind, Oxford University Press, Oxford.

Poincaré, H. (1898), "On the foundations of geometry", The Monist, 9, $1-43$.

- (1902), La Science et l'Hypothèse, Flammarion, Paris.

Pontryagin, L.S. (1946), Topological groups, Princeton University Press, Princeton.

Prauss, G. (1994), "Kant and the Straight Triangle", in Philosophy, Mathematics and Modern Physics, E. Rudolph and I.-O. Stamatescu (Eds.), Springer, Heidelberg. 226-234.

Resnik, M.D. (1975), "Mathematical Knowledge and Pattern Recognition", Canadian Journal of Philosophy, 5, 25-39.

- (1981), "Mathematics as a Science of Patterns: Ontology and Reference", Noûs, 15, 529-550.

Riemann, B. (1990), "On the Hypothesis which lie at the Basis of Geometry", in Gesammelte mathematische Werke/Collected Papers, new edition edited by R. Narasimhan, Springer, Berlin.

Roseman, D. (1997a), "On Wiener's thought on the computer as an aid in visualizing higher-dimensional forms and its modern ramifications", in V. Mandrekar and P.R. Mesani (eds.), Proceedings of Nobert Wiener Centenary Congress, PSAM, Vol. 52, American Mathematical Society, 441-471. 
- (1997b), "What Should a Surface in 4-Space Look Like?", in Visualization and Mathematics: Experiments, Simulations, Environments, H.C. Hege \& K. Polthier (eds.), Springer, Berlin, 67-82.

Rota, G.-C., D.H. Sharp, and R. Sokolowski (1998), "Syntax, Semantics, and the Problem of the Identity of Mathematical Objects", Philosophy of Science, 55, 376-386.

Rovelli, C. (1995), "Outline of a generally covariant quantum field theory and quantum theory of gravity", Journal of Mathematical Physics, 36 (1), 6529-6547.

Smale, S. (1958), "A classification of immersions of the two-sphere", Trans. Amer. Math. Soc., 90, 281-290.

Thom, R. (1990), Apologie du logos, Hachette, Paris.

- (1992), "L'Antériorité Ontologique du Continu", in J.-M. Salanskis \& H. Sinaceur (Eds.), Le Labyrinthe du Continu, Springer-Verlag, Heidelberg-Paris, 137-143.

Thurston, W.P. (1994), "On proof and progress in mathematics", Bulletin of the American Mathematical Society, 30 (2), 161-177.

Tymoczko, Th. (ed.) (1998), New Directions in the Philosophy of Mathematics, Princeton University Press, Princeton.

Suppes, P. (1977), “Is Visual Space Euclidean?”, Synthese, 35, 397-421.

Tieszen, R.L. (1989), Mathematical Intuition: Phenomenology and Mathematical Knowledge, Kluwer, Dordrecht.

Torretti, R. (1972), "On the subjectivity of objective space", in Proceedings of the Third International Kant Congress, L.W. Beck (ed.), D. Reidel, Dordrecht, 568-573.

Vuillemin, J. (1994), L'Intuitionnisme Kantien, Vrin, Paris.

Webb, J. (1987), "Immanuel Kant and the greater glory of geometry", in Naturalistic Epistemology, A. Shimony and D. Nailis (Eds.), Boston Studies in the Philosophy of Science, Vol. 100, D. Reidel, Dordrecht, 17-70.

Weyl, H. (1949), Philosophy of Mathematics and Natural Science, Princeton University Press, Princeton.

- (1973), Das Kontinuum (first German edition: 1918), Chelsea, New York. 
Wiener, N. (1922), "The relation of space and geometry to experience", The Monist, 32, 12-60; 200-247; 364-394.

Wilder, R. (1967), "The Role of Intuition", Science, 156, 605-610.

Willaschek, M. (1997), "Der transzendentale Idealismus und die Idealität von Raum und Zeit", Zeitschrift für philosophische Forschung, 51, 537-563.

Witten, E. (1988), “Topological Quantum Field Theory”, Communications in Mathematical Physics, $117,353-386$.

Wojtowicz, R. (1997), "The Metaphysical Exposition of Space and Time", Synthese, 113, 71-115.

Yang, C.N., Mills, R. (1954), "Conservation of Isotopic Spin and Isotopic Gauge Invariance, " Physical Review, 96 (1), 191-195.

Yu, T.T., Yang, C.N. (1975), "Concept of non-integrable phase factors and global formulation of gauge fields", Physical Review, D12, 3845-3857.

Zeidler, E. (2011), Quantum Field Theory III: Gauge Theory. A Bridge between Mathematicians and Physicists, Springer-Verlag; Berlin Heidelberg. 